\title{
Avalanche Behavior in an Absorbing State Oslo Model
}

\author{
Kim Christensen 7 Nicholas R. Moloney $\mathbb{A}^{7}$ and Ole Peter \\ Physics of Geological Processes, University of Oslo, PO Box 1048, Blindern, N-0316 Oslo, Norway \\ Permanent: Blackett Laboratory, Imperial College London, \\ Prince Consort Road, London SW7 2AZ, United Kingdom \\ Gunnar Pruessne1\$ \\ Physics Department, Virginia Polytechnic Inst. 8 State Univ., Blacksburg, VA 24061-0435, USA
}

(Dated: October 3, 2018)

\begin{abstract}
Self-organized criticality can be translated into the language of absorbing state phase transitions. Most models for which this analogy is established have been investigated for their absorbing state characteristics. In this article, we transform the self-organized critical Oslo model into an absorbing state Oslo model and analyze the avalanche behavior. We find that the resulting gap exponent, $D$, is consistent with its value in the self-organized critical model. For the avalanche size exponent, $\tau$, an analysis of the effect of the external drive and the boundary conditions is required.
\end{abstract}

PACS numbers: 89.75.-k, 89.75.Da, 05.65.+b, 45.70.Ht

Self-organized criticality (SOC) refers to the tendency of non-equilibrium dissipative systems, with many degrees of freedom driven at a slow rate, to display scale invariance without the fine-tuning of any control parameter [1, 2]. SOC is present in many open systems where the activity, that is, the presence of avalanches, transports slope particles through the system to the boundaries where they are eventually dissipated. When there is no activity, the system is in a so-called absorbing state. The separation of relaxational and driving timescales is achieved by adding a slope unit only when the system is in an absorbing state. Thus, the dynamics of the model implicitly tune the slope density to values that are associated with the transition between absorbing (inactive) and active states.

In a closed system, an absorbing state (AS) phase transition refers to the transition from an absorbing (inactive) state to an active state of the system at a critical value of a control parameter such as the slope density [3] .

In retrospect, Tang and Bak's 1988 description of selforganized criticality contains the ideas and features of absorbing state phase transitions [4]. This link was later clarified by Vespignani and Zapperi [5] and a recipe for transforming AS models into equivalent SOC models was devised [6]: Start with a system displaying an AS phase transition. When the control parameter is above its critical value, there is activity in the system. This activity should be coupled via the dynamics to a decrease in the control parameter, as for example when the activity reaches the boundary. Conversely, when the control parameter is below its critical value, there is no activity and the system is in an absorbing state. A process, such as

\footnotetext{
*Electronic address: k.christensen@imperial.ac.uk

${ }^{\dagger}$ Electronic address: n.moloney@imperial.ac.uk

${ }^{\ddagger}$ Electronic address: ole.peters@imperial.ac.uk

$\S$ Electronic address: gunnar.pruessner@physics.org
}

the external drive, should increase the control parameter by a small amount which may force the system into an active state. However, this picture ignores, for example, the problem of defining observables common to both AS and SOC models as well as the rôle of finite-size effects.

In the following, we transform the SOC Oslo model with one open boundary into an AS Oslo model with periodic boundary conditions. The difficulties in this procedure are shortly discussed. Extensive numerical simulations are analyzed with respect to scale invariance. The results lead to a discussion on the effect of the external drive and the boundary conditions.

The model [7] was inspired by an experiment, conducted in Oslo, on slowly driven rice-piles displaying self-organized criticality [8]. A one-dimensional lattice of length $L$ is characterized by a slope variable, $z_{i}$, and a critical slope, $z_{i}^{c}$, assigned to each site $i=1, \ldots, L$. After initialization with $z_{i}=0$ and $z_{i}^{c}$ drawn randomly with equal probability from $\{1,2\}$, the model is updated as follows: Driving: A slope unit is added to the leftmost site $i=1$, such that $z_{1} \rightarrow z_{1}+1$. Toppling: If $z_{i}>z_{i}^{c}$ at a site $i$, one slope unit is moved to each of the two nearest neighbors, that is, $z_{i} \rightarrow z_{i}-2$ and $z_{i \pm 1} \rightarrow z_{i \pm 1}+1$ except when site $i=1$ topples, where $z_{1} \rightarrow z_{1}-2$ and $z_{2} \rightarrow z_{2}+1$ or when site $i=L$ topples, where $z_{L} \rightarrow z_{L}-1$ and $z_{L-1} \rightarrow z_{L-1}+1$. After each toppling a new value for $z_{i}^{c}$ is chosen randomly with equal probability from $\{1,2\}$. The activity stops when $z_{i} \leq z_{i}^{c}$ everywhere. The model is then driven again.

After a transient, the slope density, $\zeta=(1 / L) \sum_{i=1}^{L} z_{i}$, fluctuates about a constant value. The avalanche size, $s$, is the total number of topplings after the addition of a slope unit. The avalanche size probability density function, $P(s ; L)$, in a system of size $L$ follows simple scaling above a lower cutoff $s_{\ell}$,

$$
P(s ; L)=a s^{-\tau} \mathcal{G}\left(\frac{s}{b L^{D}}\right) \text { for } s>s_{\ell},
$$

where $D$ is the gap exponent (avalanche dimension) and 
$\tau$ the avalanche size exponent. The two constants $a$ and $b$ are metric factors. The scaling function $\mathcal{G}(x)$ falls off sufficiently fast such that all moments of the avalanche size probability density function exist in a finite system. For $n>\tau-1$, the leading order of the $n$th moment is $[9]$

$$
\left\langle s^{n}\right\rangle(L)=\int_{0}^{\infty} s^{n} P(s ; L) d s=a\left(b L^{D}\right)^{n+1-\tau} g_{n}+\cdots,
$$

where $g_{n}$ depends only on the scaling function $\mathcal{G}[10$. The sub-leading terms represent, for example, corrections to scaling [11] and the presence of a lower cutoff.

The numerical estimates of the exponents for the onedimensional SOC Oslo model are very well established with $D=2.25(2)$ and $\tau=1.555(2)$ 7, 10, 12, 13, independent of the exact boundary condition at $i=L[18]$. In the following, we address the key questions: Is it possible to recover these exponents, characterizing the avalanche behavior and therefore the SOC aspect of the model, in its AS counterpart? Is the critical slope density identical in both models?

The recipe mentioned in the introduction can be "inverted" to transform the SOC Oslo model into an AS version by imposing periodic boundary conditions such that sites $i=1$ and $i=L$ are nearest neighbors, that is, for all sites toppling (including $i=1$ and $i=L$ ), one slope unit is moved to each of the two nearest neighbors. The resulting model is translationally invariant, so that the original interpretation of the slopes as height differences between columns of rice breaks down 1, 7]. The slope density increases in steps of $1 / L$ when the system is driven. If the external drive triggers activity, the avalanche will propagate until the systems falls into an absorbing state again. Contrary to the SOC Oslo model, there is no dissipation mechanism coupled to the activity to decrease the slope density in the AS Oslo model.

Every finite system will eventually fall into an absorbing state, provided that $\zeta \leq 2$. However, numerically it becomes clear that for system sizes $L \gg 1$ there exists a critical slope density, $\zeta_{c}<2$, above which the absorbing states become practically inaccessible. Defining the activity as the density of sites where $z_{i}>z_{i}^{c}$, the activity picks up sharply at the critical slope density, $\zeta_{c}$. However, one fundamental problem with a definition of an instantaneous activity is the Abelian nature of the Oslo model [14], meaning that the order in which sites are relaxed is irrelevant. Without an a priori order of relaxation, there can be no a priori microscopic timescale, and the temporal behavior of the instantaneous activity depends on the choice of microscopic timescale. It will be argued below that $\zeta_{c}$ and all relevant observables can be be obtained while avoiding these ambiguities.

Starting from an empty configuration, $\zeta=0$, slope units are added at site $i=1$ although, of course, all sites are equivalent because of translational invariance. Just as in the SOC Oslo model, slope units are added only when activity has ceased. Thus, one obtains avalanche sizes for slope densities increasing in steps of $1 / L$. At small slope densities, only small avalanches occur and until avalanches wrap around the system, the system size cannot have any effect on the dynamics. In this regime, we find that the behavior of the model essentially resembles that of the one dimensional BTW model [1] where the avalanche size scales like the square of the number of slope units added, $(\zeta L)^{2}$.

At sufficiently large slope densities, the avalanche sizes start to deviate from the above behavior. According to the original arguments [5], one expects that at the AS critical point, $\zeta_{c}$, the avalanche size probability density function follows simple scaling, Eq. (11). Let $\left\langle s^{n}\right\rangle(\zeta ; L)$ denote the $n$th moment of the avalanche size probability density function in the AS Oslo model of size $L$ at slope density $\zeta$. Ignoring corrections to scaling and the existence of a lower cutoff in Eq. (2), one expects that at $\zeta_{c}$ there exist exponents

$$
\gamma_{n}=D(n+1-\tau)
$$

such that

$$
\frac{\left\langle s^{n}\right\rangle\left(\zeta_{c} ; L\right)}{L^{\gamma_{n}}}=a b^{n+1-\tau} g_{n} \quad \text { for } L \gg 1
$$

Note that the right hand side is independent of $L$ but depends on $n$, the order of the moment. However, away from $\zeta_{c}$ the ratio on the left hand side depends on $L$. Therefore, for all $n$ and three distinct system sizes $L_{1}, L_{2}, L_{3}$, there exist a unique slope density, $\zeta_{c}$, and exponent, $\gamma_{n}$, such that

$$
\frac{\left\langle s^{n}\right\rangle\left(\zeta_{c} ; L_{1}\right)}{L_{1}^{\gamma_{n}}}=\frac{\left\langle s^{n}\right\rangle\left(\zeta_{c} ; L_{2}\right)}{L_{2}^{\gamma_{n}}}=\frac{\left\langle s^{n}\right\rangle\left(\zeta_{c} ; L_{3}\right)}{L_{3}^{\gamma_{n}}} .
$$

Graphically, $\zeta_{c}$ and $\gamma_{n}$ are determined by plotting, for each system size, the rescaled $n$th moment, $\left\langle s^{n}\right\rangle(\zeta ; L) / L^{\gamma_{n}}$, versus the slope density $\zeta$ and adjusting the exponent $\gamma_{n}$ until the graphs intersect at a single point at $\zeta_{c}$, see Fig. 1 The slope of the graphs of the resulting exponents, $\gamma_{n}$, versus the order of the moment, $n$, determines the gap exponent, $D$, while the intersection with the $n$-axis gives $\tau-1$, see Eq. (3).

Since the moments are measured for slope densities increasing in steps of $1 / L$, the location of the crossings requires interpolation between the data points. This introduces arbitrariness, which can, however, be reduced strongly by choosing system sizes which are commensurable with an estimated critical slope density such that $\zeta_{c} L \in \mathbb{N}$. In a preliminary simulation with $L=$ $1024,2048,4096,8192$ we estimated $\zeta_{c}=1.73265$ which suggests $L=1268,2536,5072,10144$ as suitable system sizes. The numerical estimates presented below refer to an exponential interpolation scheme but they are very similar to those obtained from linear interpolation. All systems were initialized with $\zeta=0$ and gradually filled to slightly above $\zeta_{c}$, where the average avalanche size becomes extremely large. In each simulation, a configuration slightly below $\zeta_{c}$ was recorded and the model driven from this configuration 100 times to improve statistics. 
For every system size, we average over approximately 2000 independent realizations starting from $\zeta=0$. Thus there is a total of approximately 200, 000 correlated realizations in the neighborhood of $\zeta_{c}$. The error bars reported below are based only on the 2000 independent realizations, thereby grossly overestimating the errors.

The numerical estimate for the critical slope density, $\zeta_{c}$, is determined as the slope density at which the graphs for different system sizes cross for the rescaled $n$th moment, see Fig. 1]

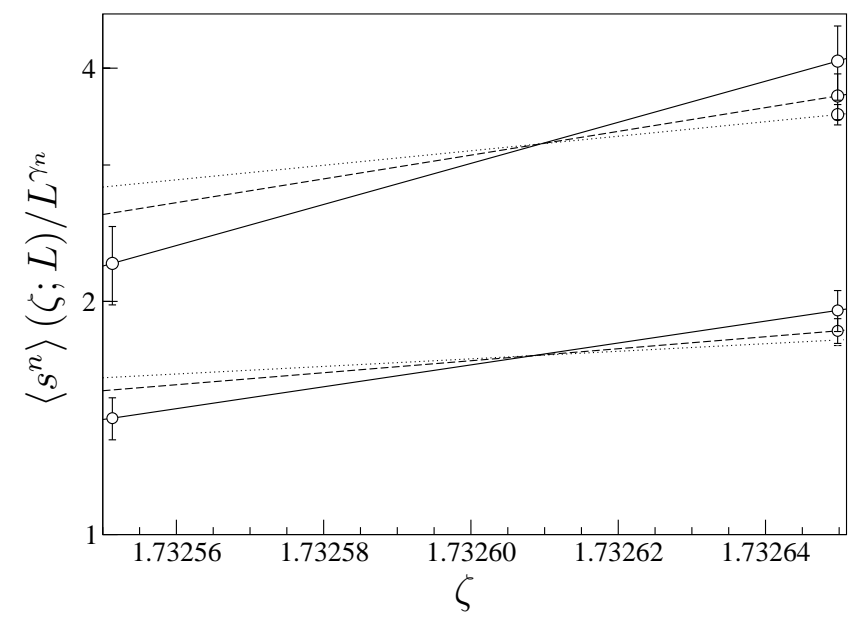

FIG. 1: The logarithm of the rescaled moments $\left\langle s^{n}\right\rangle(\zeta ; L) / L^{\gamma_{n}}$ versus the slope density $\zeta$ for $n=1,2$ (open circles). The exponential interpolations between the data points for increasing system sizes are marked with lines of increasing dash length. For the triplet $L=2536,5072,10144$, the lower set of graphs for $n=1$ intersect in a single point at $\zeta_{c}=1.732608$ with $\gamma_{1}=2.064$ and the upper set of graphs for $n=2$ intersect at $\zeta_{c}=1.732609$ with $\gamma_{2}=4.342$.

In principle, each triplet of system sizes produces an estimate of $\zeta_{c}$ for each moment. For example, from the crossings of the triplet $L=2536,5072,10144$ we find $\zeta_{c}=1.73261(5)$ for $n=1$ and $\zeta_{c}=1.73261(5)$ for $n=2$, see Fig. 11 For the triplet $L=1268,2536,5072$ the resulting estimates are $\zeta_{c}=1.73262(8)$ for $n=1$ and $\zeta_{c}=1.73259(7)$ for $n=2$. Ignoring errors, all triplets and moments yield

$$
1.73257 \leq \zeta_{c} \leq 1.73262
$$

There is no established systematic way to determine the value of $\zeta_{c}$ in the limit $L \rightarrow \infty$ but from the small change in the estimate as the system sizes are increased, it seems reasonable to estimate the critical slope density by averaging over all moments $n=1,2,3,4$ and all triplets yielding $\zeta_{c}=1.73260(2)$.

Since crossings rely on an appropriate choice of $\gamma_{n}$, estimates of the critical slope density $\zeta_{c}$ and $\gamma_{n}$ go hand in hand, see Fig. 11 Plotting $\gamma_{n}$ versus the order of the moment, $n$, produces according to Eq. (3) an estimate for $D$ and $\tau$. This procedure can be performed for every triplet of system sizes. For $L=1268,2536,5072$ we find $D=2.22(23)$ and $\tau=1.07(18)$ while for $L=2536,5072,10144$ the resulting exponents are $D=$ 2.28(23) and $\tau=1.09(13)$, see Fig. 2

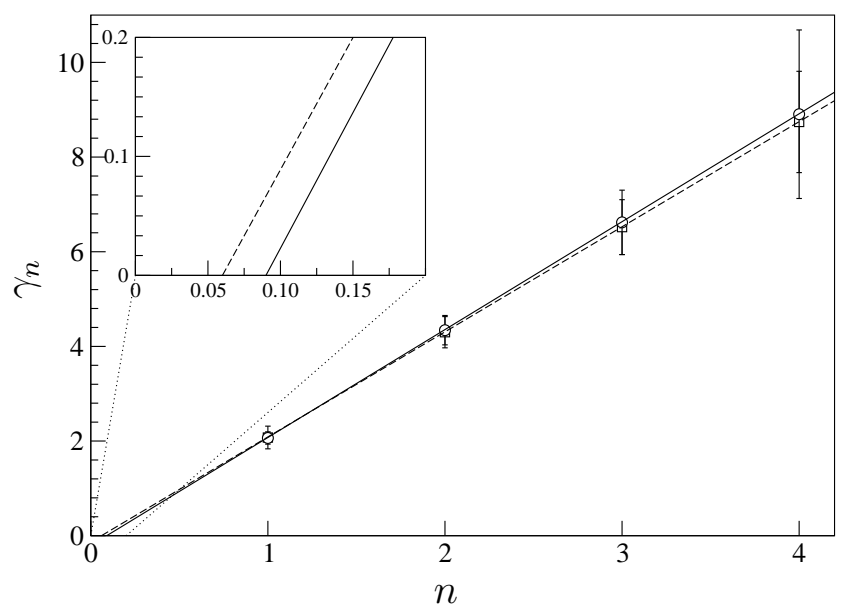

FIG. 2: The estimated exponents $\gamma_{n}$ versus the order, $n$, of the moment for the triplets $L=1268,2536,5072$ (open squares) and $L=2536,5072,10144$ (open circles). Assuming simple finite-size scaling it follows from Eq. (3) that the slope of the graphs is the gap exponent, $D$, while the intersection with the $n$-axis is $\tau-1$, see inset (dotted lines indicate magnified region). Linear regression yields $D=$ $2.22(23), \tau=1.07(18)$ for the first triplet (dashed line) and $D=2.28(23), \tau=1.09(13)$ for the second triplet (solid line).

The errors of these exponents were estimated by standard error propagation. Just as for the estimate of $\zeta_{c}$, the procedure only provides exponents for a particular set of system sizes. However, even the preliminary simulations with $L=1024,2048,4096,8192$ are fully compatible with the exponents $D$ and $\tau$ reported above. Based on all four possible triplets of system sizes, it therefore seems justified to estimate $D=2.25(8)$ and $\tau=1.08(5)$, in the limit $L \rightarrow \infty$.

Table I summarizes the numerical estimates for the critical slope densities and exponents for the AS and boundary driven SOC Oslo model.

\begin{tabular}{|l|l|c|l|c|}
\hline & \multicolumn{4}{|c|}{ Quantity } \\
\hline Oslo model & \multicolumn{1}{|c|}{$\zeta_{c}$} & $D$ & $\tau$ & $\gamma_{1}$ \\
\hline AS & $1.73260(2)$ & $2.25(8)$ & $1.08(5)$ & $2.07(6)$ \\
\hline SOC, boundary driven & $1.7326(3)$ & $2.25(2)$ & $1.555(2)$ & 1 \\
\hline SOC, bulk driven & $1.734(2)$ & $2.25(3)$ & $1.10(3)$ & 2 \\
\hline
\end{tabular}

TABLE I: The critical slope density $\zeta_{c}$, the gap exponent $D$, the avalanche size exponent $\tau$, and the exponent $\gamma_{1}$ in the AS Oslo model (all errors based on averaging over the four triplets), SOC Oslo model driven at the boundary 7,10 , [12, 13] or in the bulk [15]. For SOC Oslo models, a simple conservation argument in the stationary regime determines $\gamma_{1}$.

We are not aware of a systematic study of the average slope density $\langle\zeta\rangle(L)$ in the SOC Oslo model. How- 
ever, results from simulations of systems sizes $L=$ $1024,2048,4096,8192$ are consistent with $\zeta_{c}-\langle\zeta\rangle(L) \propto$ $L^{-x}$ with $x \approx 0.7$ and $\zeta_{c}=1.7326(3)$, where the error bar is based on visual inspection. Similarly for the bulk driven model (see below) we find $\zeta_{c}=1.734(2)$.

It is surprising how well the non-universal slope density of the SOC Oslo model is reproduced by the AS model. This, however, is exactly what is predicted by the simple mechanism put forward by Vespignani and Zapperi [5].

The avalanche size exponent $\tau=1.08(5)$ in the AS Oslo model is inconsistent with the value $\tau=1.555(2)$ in the SOC Oslo model. However, the gap exponent $D=2.25(8)$ in the AS Oslo model is consistent with the value $D=2.25(2)$ reported in the literature for the SOC Oslo model 7, 10, 12, 13]. While the former result questions the one-to-one correspondence between the SOC and AS Oslo model, one might understand this discrepancy as follows: The exponent actually characterizing the Oslo model is the gap exponent $D$. The gap exponent is deeply rooted in the model and is also present in the corresponding field theory 12, 16]. In contrast, in the SOC Oslo model, the avalanche size exponent $\tau$ is determined by the gap exponent $D$ via the scaling relation Eq. (3), since $\gamma_{1}$ can be derived by simple conservation arguments in the stationary regime; driving the SOC Oslo model at any fixed, absolute position, such as $i=1$ in the boundary driven SOC Oslo model described above, leads to $\gamma_{1}=1$, implying $D(2-\tau)=1$. Driving the model randomly in the bulk with slope units or at any relative position, such as $i=L / 2$, leads to $\gamma_{1}=2$, implying $D(2-\tau)=2$. The latter scaling law produces $\tau=1.111(8)$ from $D=2.25(2)$, well compatible with the result found in the AS Oslo model. In fact, the exponents $D=2.25(3)$ and $\tau=1.10(3)$ have been reported in the literature for a variant of the bulk-driven Oslo model [15], but interpreted as evidence against the bulk-driven SOC Oslo model being in the same universality class as the boundary driven SOC Oslo model.

Contrary to the SOC Oslo models, there exists no conservation argument to determine the value of $\gamma_{1}$ in the AS Oslo model. However, because of the periodic boundaries, the AS Oslo model seems to be related to the bulk-driven SOC Oslo model rather than the original boundary-driven SOC Oslo model. Therefore, the discrepancy in the avalanche size exponent, $\tau$, in the AS Oslo model with respect to the SOC Oslo model driven at any fixed position might very well be caused by the difference in the external drive and the resulting scaling relations. In fact, the AS Oslo model analyzed above seems to be a perfect AS version of an SOC Oslo model driven in the bulk at, say, $i=L / 2$.

Since the SOC Oslo model is equivalent to an interface depinning model [12, 16], the latter should also have an equivalent $\mathrm{AS}$ version, see discussion in Ref. 17]. It is also conjectured that the train model is in the same universality class as the Oslo model [12]. Whether imposing periodic boundary conditions on the train model will transform it into its AS analogue is an intriguing question which is beyond the scope of this brief report.

In conclusion, we have transformed the SOC Oslo model into an AS Oslo model. We have numerically determined the critical slope densities and obtained numerical estimates for the gap exponent, $D$, and avalanche size exponent, $\tau$, characterizing the avalanche behavior assuming simple finite-size scaling. The critical slope densities and the gap exponents are identical in the two models. However, the avalanche size exponents are different. The question remains whether one can construct a "proper" AS Oslo model, corresponding to the boundary driven SOC Oslo model.

The authors wish to thank A. Thomas as well as D. Moore, B. Maguire and P. Mayers for their technical support of the SCAN cluster at the Department of Mathematics at Imperial College London. The authors are indebted to PGP, University of Oslo, Norway for support and hospitality during their visit. NRM is very grateful to the Beit Fellowship. OP and GP gratefully acknowledge the support of EPSRC, GP also acknowledges the support of the AvH Foundation and the NSF.
[1] P. Bak, C. Tang, and K. Wiesenfeld, Phys. Rev. Lett. 59, 381 (1987).

[2] H. J. Jensen, Self-Organized Criticality (Cambridge University Press, New York, NY, 1998).

[3] H. Hinrichsen, Adv. Phys. 49, 815 (2000), condmat/0001070v2.

[4] C. Tang and P. Bak, J. Stat. Phys. 51, 797 (1988).

[5] A. Vespignani and S. Zapperi, Phys. Rev. Lett. 78, 4793 (1997).

[6] R. Dickman, M. A. Muñoz, A. Vespignani, and S. Zapperi, Braz. J. Phys. 30, 27 (2000), cond-mat/9910454v2.

[7] K. Christensen, A. Corral, V. Frette, J. Feder, and T. Jøssang, Phys. Rev. Lett. 77, 107 (1996).

[8] V. Frette, K. Christensen, A. Malthe-Sørenssen, J. Feder, T. Jøssang, and P. Meakin, Nature 379, 49 (1996).

[9] M. De Menech, A. L. Stella, and C. Tebaldi, Phys. Rev. E
58, R2677 (1998).

[10] G. Pruessner and H. J. Jensen, Phys. Rev. Lett. 91, 244303 (2003), cond-mat/0307443.

[11] F. J. Wegner, PRB 5, 4529 (1972).

[12] M. Paczuski and S. Boettcher, Phys. Rev. Lett. 77, 111 (1996).

[13] K. Christensen (2004), in press, Per Bak memorial issue, Physica A 4130, available online June 16.

[14] D. Dhar (2003), preprint cond-mat/0309490.

[15] A. Malthe-Sørenssen, Phys. Rev. E 59, 4169 (1999).

[16] G. Pruessner, Phys. Rev. E 67, 030301(R) (2003), condmat/0209531.

[17] G. Pruessner, Ph.D. thesis, Imperial College London, 180 Queen's Gate, London SW7 2BZ, UK (2004).

[18] The simulations in Ref. [10] refer to a boundary condition where site $i=L$ relaxes according to $z_{L} \rightarrow z_{L}-2$ and 
$z_{L-1} \rightarrow z_{L-1}+1$. 